\title{
Quantitative Evaluation of Aerosol Generation from Non-contact Tonometry and its Correlation with Tear Film Characteristics
}

\author{
Weiting Hao $\cdot$ Jianhui $\mathrm{Wu} \cdot$ Xinheng Zhao $\cdot$ Danni Liang $\cdot$ \\ Xingchen $\mathrm{Yu} \cdot$ Huazheng Cao $\cdot$ Yan Wang (D)
}

Received: March 6, 2021 / Accepted: April 7, 2021 / Published online: April 28, 2021

(c) The Author(s), under exclusive licence to Springer Healthcare Ltd., part of Springer Nature 2021

\begin{abstract}
Introduction: Ophthalmologists are inevitably exposed to tears and ocular discharge during ophthalmologic examinations and are at high risk for SARS-CoV-2 infection. To understand the role of aerosols in disease transmission, we adopted a prospective cross-sectional study design and investigated the count and size distribution of aerosols generated by a non-contact tonometer and its correlation with individual tear film characteristics.

Methods: This study constituted two parts. The study population included outpatients who
\end{abstract}

W. Hao $\cdot$ X. Zhao $\cdot$ X. Yu $\cdot$ H. Cao $\cdot$ Y. Wang Clinical College of Ophthalmology, Tianjin Medical University, Tianjin, China

J. $\mathrm{Wu}$

State EnvironmentalProtection Key Laboratory of

Urban Ambient Air Particulate Matter Pollution

Prevention and Control, College of

EnvironmentalScience and Engineering, Nankai

University, Tianjin, China

Y. Wang $(\bowtie)$

Tianjin Eye Hospital, Tianjin Eye Institute, Tianjin

Key Laboratory of Ophthalmology and Visual

Science, Clinical College of Ophthalmology, Tianjin

Medical University, No 4. Gansu Road, Heping

District, Tianjin 300020, China

e-mail: wangyan7143@vip.sina.com

D. Liang

Tianjin Shuangyun Environmental Protection

Technology Co., Ltd, Tianjin, China underwent an intraocular pressure examination in an intraocular pressure examination room (Part I) and 20 participants who underwent an intraocular pressure examination in a laboratory (Part II). The following main outcomes were measured: aerosol counts at 0, 50, 100, 150 , and $200 \mathrm{~cm}$ from the non-contact tonometer (Part I); aerosol counts after each participant underwent non-contact tonometry, and lipid layer thickness score and tear film break-up time (Part II).

Results: The aerosol count decreased with increasing distance from the tonometer. The aerosol count at $0 \mathrm{~cm}$ had the highest value compared to that at other distances. For aerosols of diameters $0.25-0.5 \mu \mathrm{m}$ and $0.5-1.0 \mu \mathrm{m}$, the count decreased at $50 \mathrm{~cm}$ and remained stable at further distances. For aerosols of diameters $1.0-2.5 \mu \mathrm{m}$ and $\geq 2.5 \mu \mathrm{m}$, the count dropped progressively at all five distances. The aerosol count from each tonometer correlated positively with the lipid layer thickness score $(r=0.490, \quad P=0.028)$, whereas the aerosol count correlated negatively with the tear film break-up time $(r=-0.675, P=0.001)$.

Conclusions: Aerosols tended to coagulate during diffusion. A $50-\mathrm{cm}$ distance from the tonometer could confer safety from aerosols with $<1.0-\mu \mathrm{m}$ diameter. Aerosols generated during non-contact tonometry could contain a lipid layer component. Moreover, tear film stability could affect aerosol generation. Protective eyewear is recommended for reducing infection 
risk from aerosols. Individual tear film characteristics should be considered during non-contact tonometry.

Keywords: Ophthalmology; Non-contact tonometry; SARS-CoV-2; COVID-19; Aerosols; Tear film

\section{Key Summary Points}

Why carry out this study?

Aerosols generated during ophthalmology procedures, such as non-contact tonometry, may contaminate the human conjunctival epithelium

SARS-CoV-2 has the potential to be transmitted via aerosols posing a risk for ophthalmologists

What was the hypothesis of the study?

We studied the generation and distribution of aerosols from non-contact tonometry and the impact of tear film characteristics on aerosol generation

\section{What was learned from the study?}

A $50 \mathrm{~cm}$ distance from the tonometer may confer safety from aerosols with $<1.0-\mu \mathrm{m}$ diameter

Patients with aqueous-deficient dry eyes will tend to generate more aerosols, and the use of protective eyewear in clinical settings for both doctors and patients is recommended

\section{DIGITAL FEATURES}

This article is published with digital features, including a summary slide, to facilitate understanding of the article. To view digital features for this article go to https://doi.org/10.6084/ m9.figshare.14376944.

\section{INTRODUCTION}

Coronavirus disease 2019 (COVID-19) is spreading worldwide, and the causative pathogen has been identified as severe acute respiratory syndrome coronavirus 2 (SARS-CoV-2) $[1,2]$. A study has found that despite respiratory protection, ophthalmologists still possess a high risk for infection [3]. During the earlier period of the COVID-19 outbreak, Cheng et al. [4] proposed that SARS-CoV-2 could contaminate the human conjunctival epithelium, leading to respiratory infection. Sergio et al. [5] reported that conjunctivitis could be the only presenting sign or symptom of COVID-19, and Marinho et al. [6] described retinal findings in such patients. However, the mechanisms underlying the risk of infection to ophthalmologists remain unclear.

SARS-CoV-2 has the potential to be transmitted via aerosols; Liu et al. [7] detected SARSCoV-2 RNA in aerosols in different areas of two Wuhan hospitals during the COVID-19 outbreak. Recently, it has been shown that aerosols are generated during ophthalmology procedures such as non-contact tonometry, and endoscopic dacryocystorhinostomy and with the use of lasers, electrical cutting, and coagulation systems during surgery [8-11]. Furthermore, several studies [12-14] have provided evidence that aerosol transmission is an important route of disease communication, thereby providing significant insight in understanding the transmission and control of SARSCoV-2.

The non-contact tonometer, widely used in ophthalmology clinics, works by utilizing a brief pulse of pressurized air. However, Britt et al. [15] reported that the eyes reveal some degree of tear film dehiscence and micro-aerosol formation in association with non-contact tonometry, and the possibility of viruses being present in these droplets is concerning. Previous studies [16-18] have found evidence of viruses (such as human immunodeficiency virus, Epstein-Barr virus, and measles virus) in tears. Recently, the presence of SARS-CoV-2 in ocular samples (tears and conjunctival swabs) has also been reported [19]. The presence of the SARS- 
CoV-2 receptor in the eye could explain the viral tropism in the ocular tissue [20-22]. Bearing in mind the possibility of viruses (particularly SARS-CoV-2) in aerosols from ocular samples, the generation of aerosols from noncontact tonometry should be considered as an important risk factor for ophthalmology work environment.

Adverse effects of aerosols on human health can be correlated with their size distribution, as the particle size of aerosols affects their dynamics and deposition [23]. Nevertheless, there are few reports on the size distribution of aerosols using non-contact tonometry. It is speculated that aerosols are generated from the tear film dehiscence [15]. Many viruses, including SARS-CoV-2, are covered with a lipid bilayer envelope, which could affect the survival and invasion pathways of viruses [24-27]. The lipid layer is a part of the tear film, and we hypothesized that the lipid droplets in this layer could affect the colonization and release of viruses. Hence, we considered it necessary to investigate the relationship between aerosol generation and lipid layer thickness. In addition, the stability of the tear film could also contribute to tear film dehiscence from noncontact tonometry; therefore, in this study, we also focused on the tear film break-up time (TBUT), which is one of the indexes used to evaluate tear film stability.

In this study, our objective was to investigate the count and size distribution of aerosols from non-contact tonometry and explore the impact of tear film characteristics on aerosol generation. This work highlights the urgent need to clarify the relationship between aerosol generation and ophthalmology procedures and guide the formulation of clinical protection principles to prevent the further spread of COVID-19.

\section{METHODS}

The study protocol adhered to the tenets of the Declaration of Helsinki and was approved for measurement and data analysis by the Ethics Committee of Tianjin Eye Hospital, Tianjin, China (no. 202031). Informed consent was obtained from all participants.
This prospective study comprised two parts. Part I was conducted in the intraocular pressure (IOP) examination room. Patients aged 18-65 years underwent non-contact tonometry as outpatients. The exclusion criterion was the presence of active inflammatory ocular diseases, defined as any ocular disease that was not suitable for non-contact tonometry, such as ocular diseases caused by bacteria or viruses (e.g., conjunctivitis and keratitis). A portable optical particle size spectrometer (11-A; GRIMM, Ainring, Germany) provided an instantaneous count of aerosols every $6 \mathrm{~s}$. The aerosols were divided into four segments according to their diameters. The aerosol diameters for segments $\mathrm{A}, \mathrm{B}, \mathrm{C}$, and $\mathrm{D}$ were $0.25 \mu \mathrm{m} \leq x<0.5 \mu \mathrm{m}$, $0.5 \mu \mathrm{m} \leq x<1.0 \mu \mathrm{m}, 1.0 \mu \mathrm{m} \leq x<2.5 \mu \mathrm{m}$, and $2.5 \mu \mathrm{m} \leq x$, respectively. The total aerosol count was also recorded. The count of aerosols was evaluated at $0 \mathrm{~cm}, 50 \mathrm{~cm}, 100 \mathrm{~cm}, 150 \mathrm{~cm}$, and $200 \mathrm{~cm}$ from the non-contact tonometer. In each examination, the spectrometer was allowed to sit for at least $10 \mathrm{~min}$ until the reading was stable, and then the number of aerosols was recorded for $30 \mathrm{~min}$. The examinations were conducted at each site for 3 consecutive days, and average values were both calculated and defined as the count of aerosols at each site. The ambient temperature, relative humidity, space volume, and visitor flow rate of the IOP examination room were $26.13^{\circ} \mathrm{C}$, $56.05 \%, 27.07 \mathrm{~m}^{3}$, and 22 persons $/ 30 \mathrm{~min}$, respectively.

Part II was conducted in a laboratory setting after standard laminar flow purification. The purpose of purification was to eliminate the influence of aerosols in the natural air as much as possible, allowing for an easier investigation of changes in the aerosol count for each measurement. The baseline aerosol value was 264 without non-contact tonometry.

Twenty participants aged 18-65 years underwent non-contact tonometry, and the average aerosol count at $0 \mathrm{~cm}$ from the noncontact tonometer was recorded during each examination using a portable optical particle size spectrometer. Patients with active inflammatory ocular diseases, defined as any ocular disease that was not suitable for non-contact tonometry, such as ocular diseases caused by 
bacteria or viruses (e.g., conjunctivitis and keratitis), were excluded. Each participant entered the laboratory wearing a clean surgical gown. The lipid layer thickness and TBUT were evaluated for each participant.

All examinations were performed by the same technician. The lipid layer thickness score was evaluated noninvasively using a LipiView ${ }^{\circledR}$ II ocular surface interferometer (Johnson \& Johnson, New Brunswick, NJ, USA). The participant was asked to blink naturally and look into the camera; a $20 \mathrm{~s}$ video of the interference pattern of the tear film was then recorded. The illumination area for specular reflection detection is located $1 \mathrm{~mm}$ above the tear meniscus, slightly below the inferior margin of the pupil, and is limited to approximately $2.5 \mathrm{~mm}$ vertically and $5.0 \mathrm{~mm}$ horizontally. The lipid layer thickness score was quantified according to the dominant colors of the interference pattern in the area of specular reflection during the examination period. Stability and reliability were indicated as index C-factors. Data with a C-factor of $>80 \%$ qualified for inclusion in the study. This procedure has been described previously in detail $[28,29]$.

In the TBUT examination, $1 \mathrm{mg}$ fluorescein sodium ophthalmic strips (Liaoning Meizilin Pharmaceutical Corp., Liaoning, China) were moistened and placed on the lateral third of the lower eyelid, and the tear film was stained. The interval between the last complete blink and the appearance of the first dry spots on the cornea was evaluated three times, and the average value was calculated.

All data were collated and calculated using Microsoft Excel 2007 (Microsoft Corp., Redmond, WA, USA), and statistical analyses were performed using IBM SPSS Statistics for Windows (version 23.0; IBM Corp., Armonk, NY, USA). A Spearman correlation analysis was used to determine the correlation between the count of aerosols generated from non-contact tonometry and tear film-related parameters. Statistical significance was set at $P<0.05$.

\section{RESULTS}

A high-speed camera was used to capture the moment after the non-contact tonometry. Figure 1 illustrates the stained aerosol particles generated around the eye immediately after tonometry; the tonometry also simulated the dispersion of aerosol particles in the surrounding environment and increased the risk of virus transmission.

\section{Part I: Count and Size Distribution of Aerosols from Non-contact Tonometry}

The count and size distribution of aerosols at different distances from the non-contact tonometer were investigated.

Total Count of Aerosols at Different Distances The total count of aerosols decreased gradually as the distance from the tonometer increased. The total count of aerosols was 121,689, $102,537,102,486,104,837$, and 100,860 at $0 \mathrm{~cm}, 50 \mathrm{~cm}, 100 \mathrm{~cm}, 150 \mathrm{~cm}$, and $200 \mathrm{~cm}$ from the non-contact tonometer, respectively.

\section{Size Distribution of Aerosols at Different Distances}

Count of Aerosols in Four Diameter Segments at Different Distances Figure 2 illustrates the count of aerosols for four diameter segments at different distances from the non-contact

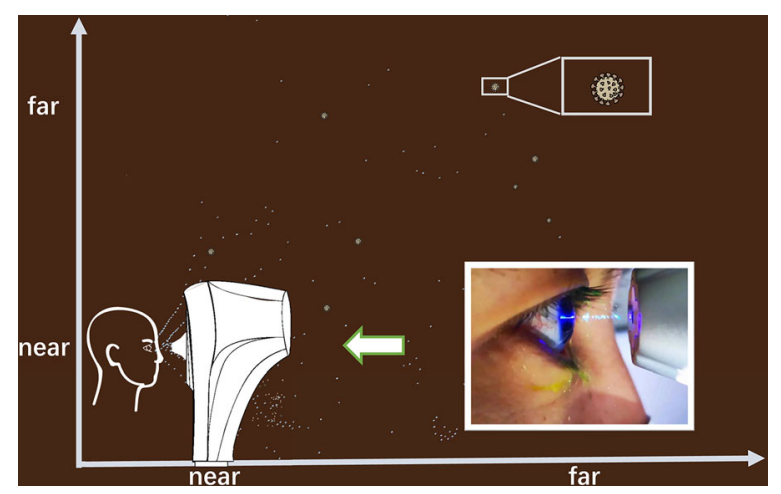

Fig. 1 Aerosol particle generation after tonometry. Stained aerosol particles generated around the eye immediately after tonometry dispersing into the surrounding environment 


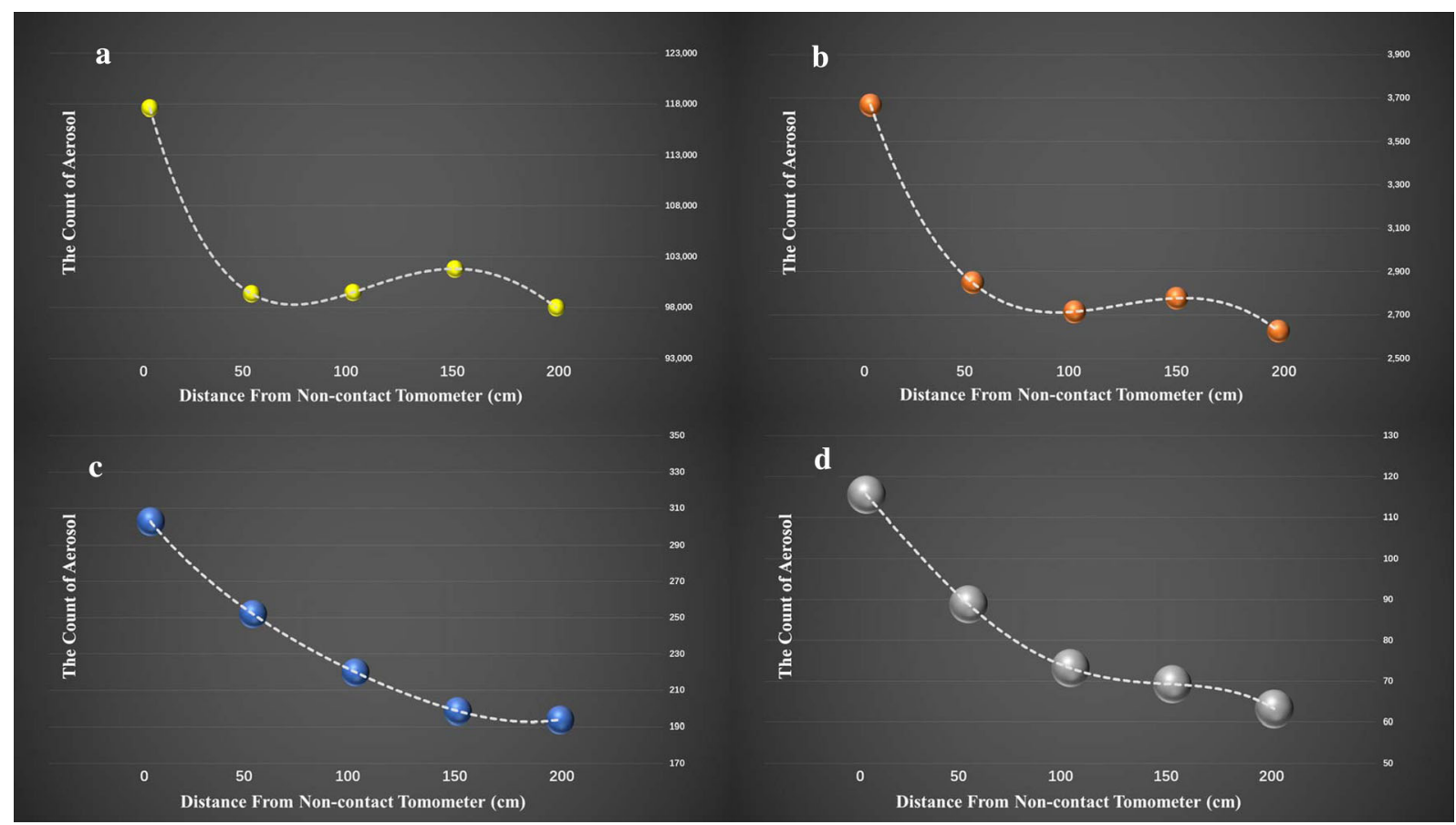

Fig. 2 Aerosol counts for four diameter segments at different distances from the non-contact tonometer. a-d Aerosol counts for segments A, B, C, and D, respectively. More aerosols are detected closer to the non-contact tonometer. For aerosols with smaller sizes (segments $\mathrm{A}$ and $\mathrm{B}$ ), the count decreases at $50 \mathrm{~cm}$ and remains relatively stable at further distances. For aerosols with larger sizes (segments $\mathrm{C}$ and $\mathrm{D}$ ), the count decreases continuously with an increase in distance

Table 1 Percentage reduction in the aerosol count between two adjacent distances

\begin{tabular}{ccccc}
\hline & Segment A (\%) & Segment B (\%) & Segment C (\%) & Segment D (\%) \\
\hline $0-50 \mathrm{~cm}$ & 15.52 & 22.33 & 16.81 & 23.12 \\
$50-100 \mathrm{~cm}$ & -0.13 & 4.71 & 12.71 & 17.62 \\
$100-150 \mathrm{~cm}$ & -2.33 & -2.29 & 9.72 & 5.40 \\
$150-200 \mathrm{~cm}$ & 3.75 & 5.44 & 2.40 & 8.66 \\
\hline
\end{tabular}

The aerosol diameters for segments A, B, C, and D are $0.25 \mu \mathrm{m} \leq x<0.5 \mu \mathrm{m}, 0.5 \mu \mathrm{m} \leq x<1.0 \mu \mathrm{m}, 1.0 \mu \mathrm{m} \leq x<2.5 \mu \mathrm{m}$, and $2.5 \mu \mathrm{m} \leq x$, respectively

tonometer. Table 1 shows the percentage reduction in the aerosol count between every two adjacent distances. For any segment, the count of aerosols at $0 \mathrm{~cm}$ (closest to the eyes) demonstrated the highest value. However, for segments A and B (Fig. 2a, b), the count of aerosols decreased at $50 \mathrm{~cm}$ and remained relatively stable at further distances. For segments C and D (Fig. 2c, d), the aerosol count dropped continuously among the five distances. This demonstrated that the size of an aerosol particle could influence its aerodynamic properties.

Percentage of the Aerosols for Four Segments in the Total Count As shown in Fig. 3, the percentages of aerosols for the four segments in the total count were compared among the five distances. For segment $\mathrm{A}$, the percentage of 


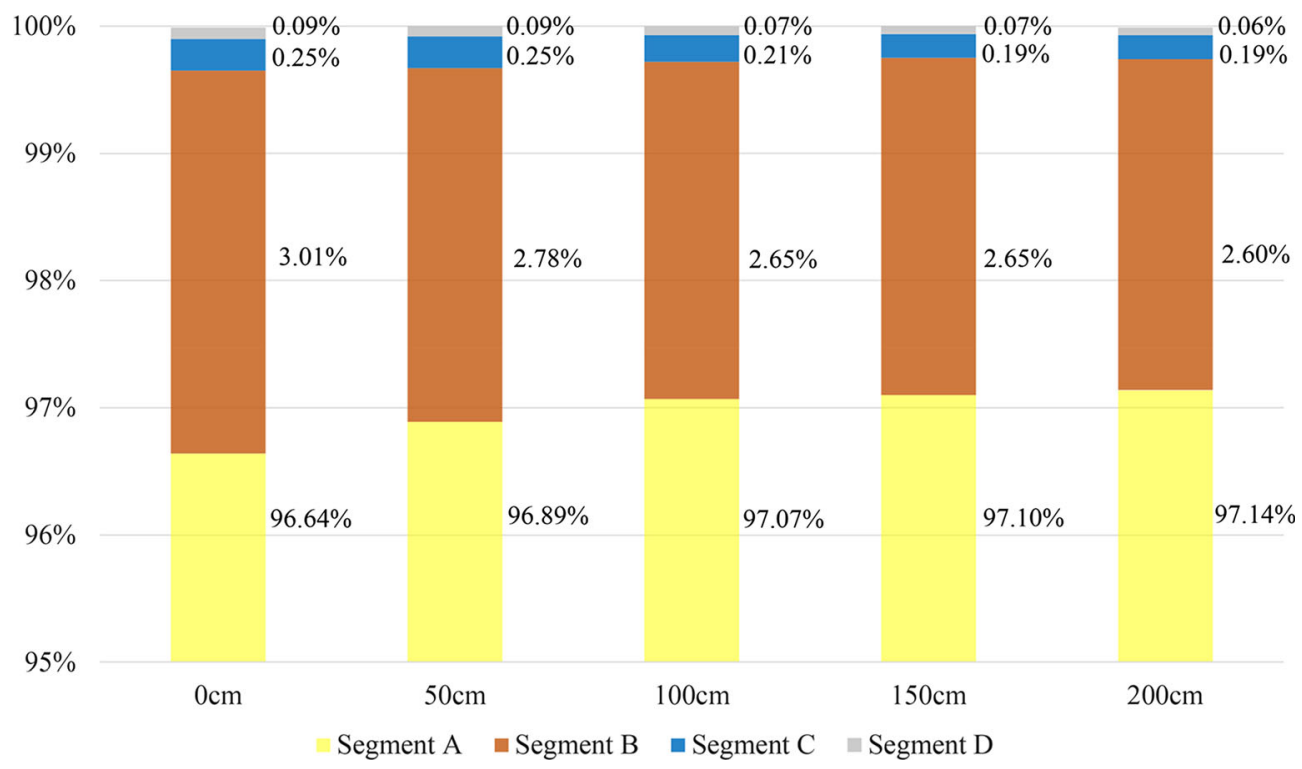

Fig. 3 Percentage of aerosols for the four segments at different distances from the non-contact tonometer. The percentage of aerosols in segment A gradually increases as

aerosols increased gradually from 96.64 to $97.14 \%$ as the distance from the tonometer increased, whereas an opposite trend was observed for segments B, C, and D. This indicates that the aerosols were composed differently at different distances.

\section{Part II: Effects of Characteristics of the Tear Film on Aerosol Generation from Non-contact Tonometry}

The correlation between the count of aerosols generated from non-contact tonometry and two tear film-related parameters (lipid layer thickness score and TBUT) was analyzed. The

Table 2 Demographic characteristics of 20 participants

\begin{tabular}{ll} 
Sex, $n(\%)$ & \\
Male & $9(45 \%)$ \\
Female & $11(55 \%)$ \\
Age (years) & $24.4 \pm 1.9$ \\
Intraocular pressure (mmHg) & $15.6 \pm 1.8$ \\
\hline
\end{tabular}

the distance increases, whereas an opposite trend is observed for segments B, C, and D

demographic data of the 20 participants are shown in Table 2.

\section{Correlation Between the Count of Aerosols and Lipid Layer Thickness Score}

The average binocular lipid layer thickness score was calculated and used to represent the thickness of the lipid layer. Figure 4 a shows the statistically significant positive correlations between the count of aerosols generated from each non-contact tonometry and the lipid layer thickness score $(r=0.549, P=0.012$ for the count of segment A; $r=0.490, P=0.028$ for the total aerosol count). Thus, participants who demonstrated higher lipid layer thickness scores tended to generate more aerosols from noncontact tonometry.

\section{Correlation Between the Count of Aerosols and Tear Film Break-up Time}

The average value of binocular TBUT was calculated and used as a parameter representing the stability of the tear film. As shown in Fig. $4 \mathrm{~b}$, statistically significant negative correlations were observed between the count of aerosols generated from each non-contact tonometry and TBUT values $(r=-0.672$, 


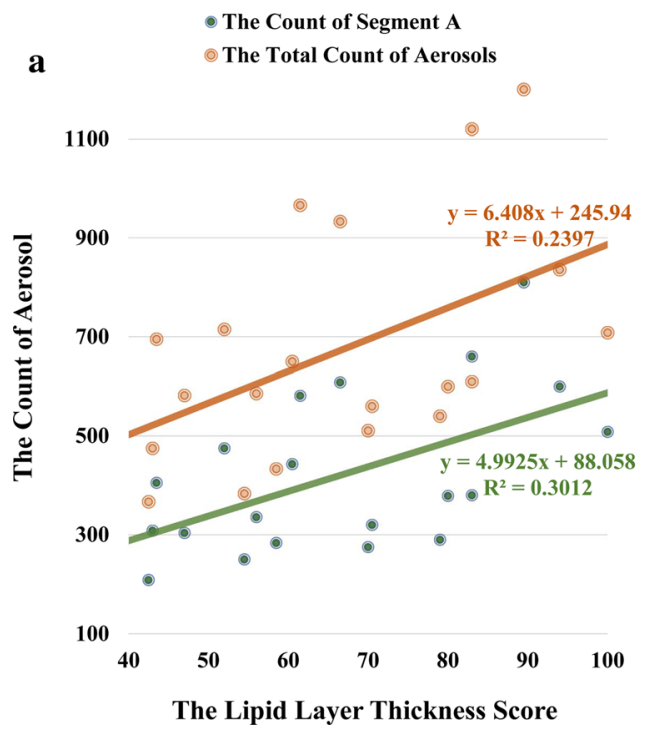

Fig. 4 Correlations between aerosol generation and characteristics of the tear film. a Correlations between the aerosol count and lipid layer thickness score. Statistically significant positive correlations are shown (green line: correlation between the count of segment $\mathrm{A}$ and lipid layer thickness score; orange line: correlation between the total

$P=0.001$ for the count of segment A; $r=-0.675, P=0.001$ for the total aerosol count). Thus, participants who demonstrated a shorter TBUT tended to generate more aerosols from non-contact tonometry. The stability of the tear film was a factor that affected the generation of aerosols.

\section{DISCUSSION}

Due to the proximity required for ophthalmic examinations and the inevitable exposure to tears and ocular discharge, ophthalmologists are at high risk for SARS-CoV-2 infection [30]. Aerosols are generated during examinations based on air pulses. We conducted this study to identify ways of minimizing the risk of SARS$\mathrm{CoV}-2$ transmission during ophthalmic examinations.

In this study, the count and size distribution of aerosols around a non-contact tonometer were investigated in an IOP examination room. We also observed correlations between the aerosol count generated from non-contact

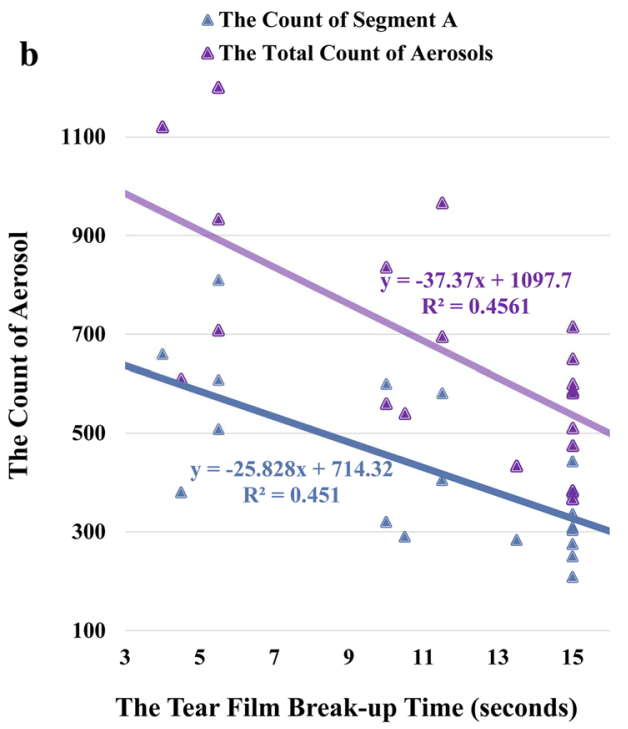

count of aerosols and lipid layer thickness score). b Correlations between the aerosol count and tear film break-up time (TBUT). Statistically significant negative correlations are shown (blue line: correlation between the count of segment $A$ and TBUT; purple line: correlation between the total aerosol count and TBUT)

tonometry and the characteristics of the tear film (lipid layer thickness score and TBUT).

The total aerosol counts gradually decreased as the distance from the tonometer increased. To ensure rigor in the experimental data, we created the following restrictions: first, all participants wore masks during the examination to reduce the impact of breathing on the aerosol count. Second, only one participant was allowed to enter the examination room for each measurement, which minimized the impact of the participants' motion. Our study confirmed that there is indeed aerosol generation from non-contact tonometry and that deposition occurs as the distance increases $[15,31]$.

The size distribution of the aerosols was as follows: the count of aerosols with small sizes (segments $\mathrm{A}$ and $\mathrm{B}$ ) decreased at $50 \mathrm{~cm}$ and remained relatively stable at further distances, whereas the count of aerosols with large sizes (segments C and D) showed an approximate linear decline with distance. The development of dynamic models of aerosols generated from non-contact tonometry requires further investigation using biological and physical methods. 
Nevertheless, we can speculate on the particle characteristics of these aerosols. Both small and large aerosols were generated using non-contact tonometry because the aerosol count was higher than that of the natural air. Aerosols tend to coagulate during diffusion [32]. For aerosols with diameters $<1 \mu \mathrm{m}$, coagulation mainly occurred within $50 \mathrm{~cm}$. It is difficult for a coagulated aerosol to diffuse. When the diameter of a coagulated aerosol is large, it will deposit on the ground or the surface of the object and therefore cannot be detected [31]. However, aerosols with large diameters $(>1 \mu \mathrm{m})$ coagulated and deposited progressively with increasing distance. Studies have shown that the diameter of aerosol particles could affect their deposition in the respiratory tract [33-35]. Considering the possibility that aerosol particles carry viruses, further experiments are needed to determine whether virus-carrying aerosol particles of different sizes have different abilities to bind to receptors on the conjunctiva. Therefore, these findings suggest that distance control could be an important factor in avoiding infection by viruses through aerosols. We speculated that $50 \mathrm{~cm}$ from the tonometer could be the relatively safe distance for aerosols with diameters $<1 \mu \mathrm{m}$. For larger aerosols, further experimental evidence is required to determine the relatively safe distance.

Additionally, the percentage of aerosols in different segments could indicate their composition at different distances. In this study, we believe that the aerosols at a distant site were more similar to those of natural air. The percentage of aerosols with smaller sizes at $0 \mathrm{~cm}$ was lower than that at $200 \mathrm{~cm}$, whereas the percentage of aerosols with larger sizes at $0 \mathrm{~cm}$ was higher than that at $200 \mathrm{~cm}$. We aim to conduct further experiments, such as scanning electron microscopy and high-throughput sequencing, to explore the biological composition of aerosols generated from non-contact tonometry.

We found that participants with a higher lipid layer thickness score and shorter TBUT tended to generate more aerosols from noncontact tonometry. The tear film is composed of a mucous layer, a watery layer, and a lipid layer. The lipid layer score is related to the meibomian gland function and correlates with dry eye symptoms [29, 36, 37]. Participants with a thicker lipid layer could be more likely to generate aerosols. Hence, we speculated that aerosols generated from non-contact tonometry contained a component of the lipid layer. Once a virus is attached to the carrier in the lipid layer, it may be possible to transfer it to the aerosols. Conversely, a shorter TBUT indicated lower stability of the tear film. It may be easier to generate aerosols when the tear film is less stable. Therefore, we hypothesize that patients with aqueous-deficient dry eye tend to generate more aerosols compared with other types of dry eye disease because they demonstrate relatively normal lipid layer thickness, while the tear film is less stable [38].

There were several limitations to the current study. First, due to the limited size of the IOP examination room, we only selected five distance sites to the tonometer for the aerosol count evaluation. Further finer site distances could provide additional information on the relatively safe distance. Second, the composition of aerosol particles generated during noncontact tonometry is still unclear. Further experiments using scanning electron microscopy and high-throughput sequencing are required. Third, further experiments are needed to determine whether high IOP affects the results.

\section{CONCLUSIONS}

We conducted this study in the context of the ongoing COVID-19 pandemic. Aerosols generated from non-contact tonometry tend to coagulate during diffusion. For aerosols with diameters $<1 \mu \mathrm{m}, 50 \mathrm{~cm}$ to the tonometer could be a relatively safe distance. Distance control could be helpful in the prevention of infection. In addition, aerosols generated from non-contact tonometry could contain a component of the lipid layer, and the stability of the tear film may also affect aerosol generation. Patients with aqueous-deficient dry eye tend to generate more aerosols than those with other types of dry eye disease do. This study highlights the importance of using protective 
eyewear in the clinical setting for both doctors and patients and paying attention to certain eye conditions (such as dry eye disease) during daily practice.

\section{ACKNOWLEDGEMENTS}

Funding. This work was supported by the National Nature Science Foundation of China (grant no. 81873684). This organization was not involved in the design of the study, collection, analysis, interpretation of data, or writing and Rapid Service Fee for the publication of the research.

Medical Writing and Editorial Assistance. We would like to thank Editage (http:// www.editage.cn) for the English language editing service.

Authorship. All named authors meet the International Committee of Medical Journal Editors (ICMJE) criteria for authorship for this article, take responsibility for the integrity of the work as a whole, and have given their approval for this version to be published.

Author's Contributions. YW, WH, and JW designed the study. $\mathrm{WH}, \mathrm{XZ}, \mathrm{XY}, \mathrm{HC}$, and $\mathrm{DL}$ collected and analyzed the data. WH wrote the original manuscript. YW and JW made revisions to the manuscript. All authors read and approved the final manuscript.

Disclosures. Weiting Hao, Jianhui Wu, Xinheng Zhao, Danni Liang, Xingchen Yu, Huazheng Cao, and Yan Wang declare they have nothing to disclose.

Compliance with Ethics guidelines. The study protocol adhered to the tenets of the Declaration of Helsinki and was approved for measurement and data analysis by the Ethics Committee of Tianjin Eye Hospital, Tianjin, China (no. 202031). Informed consent was obtained from all participants.
Data Availability. The datasets generated and analyzed during the current study are not publicly available at this time as the data also forms part of an ongoing study.

\section{REFERENCES}

1. Coronaviridae Study Group of the International Committee on Taxonomy of Viruses. The species severe acute respiratory syndrome-related coronavirus: classifying 2019-nCoV and naming it SARSCoV-2. Nat Microbiol. 2020;5:536-44.

2. Zhou P, Yang XL, Wang XG, et al. A pneumonia outbreak associated with a new coronavirus of probable bat origin. Nature. 2020;579:270-3.

3. Qiao C, Zhang H, He M, et al. Symptomatic COVID19 in eye professionals in Wuhan. China Ophthalmol. 2020;127:1268-70.

4. Lu CW, Liu XF, Jia ZF. 2019-nCoV transmission through the ocular surface must not be ignored. Lancet. 2020;395:e39.

5. Scalinci SZ, Trovato BE. Conjunctivitis can be the only presenting signs and symptoms of COVID-19. IDCases. 2020;20:e00774.

6. Marinho PM, Marcos AAA, Romano AC, Nascimento H, Belfort R Jr. Retinal findings in patients with COVID-19. Lancet. 2020;395:1610.

7. Liu Y, Ning Z, Chen Y, et al. Aerodynamic analysis of SARS-CoV-2 in two Wuhan hospitals. Nature. 2020;582:557-60.

8. Sun CB, Wang YY, Liu GH, Liu Z. Role of the eye in transmitting human coronavirus: what we know and what we do not know. Front Public Health. 2020;8:155.

9. Workman AD, Jafari A, Welling DB, et al. Airborne aerosol generation during endonasal procedures in the era of COVID-19: risks and recommendations. Otolaryngol Head Neck Surg. 2020;163:465-70.

10. Thamboo A, Lea J, Sommer DD, et al. Clinical evidence based review and recommendations of aerosol-generating medical procedures in otolaryngology — head and neck surgery during the COVID-19 pandemic. J Otolaryngol Head Neck Surg. 2020;49:28.

11. Karjalainen M, Kontunen A, Saari S, et al. Characterization of surgical smoke from various tissues and their implications for occupational safety. PLoS ONE. 2018;13:e0195274. 
12. Anderson EL, Turnham P, Griffin JR, Clarke CC. Consideration of aerosol transmission for COVID19 and public health. Risk Anal. 2020;40:902-7.

13. Mick P, Murphy R. Aerosol-generating otolaryngology procedures and the need for enhanced PPE during the COVID-19 pandemic: a literature review. J Otolaryngol Head Neck Surg. 2020;49:29.

14. Jayaweera M, Perera H, Gunawardana B, Manatunge J. Transmission of COVID-19 virus by droplets and aerosols: a critical review on the unresolved dichotomy. Environ Res. 2020;188:109819.

15. Britt JM, Clifton BC, Barnebey HS, Mills RP. Microaerosol formation in noncontact 'air-puff' tonometry. Arch Ophthalmol. 1991;109:225-8.

16. Han $\mathrm{Y}, \mathrm{Wu} \mathrm{N}, \mathrm{Zhu} \mathrm{W}$, et al. Detection of HIV-1 viruses in tears of patients even under long-term HAART. AIDS. 2011;25:1925-7.

17. Willoughby CE, Baker K, Kaye SB, et al. Epstein-Barr virus (types 1 and 2) in the tear film in Sjogren's syndrome and HIV infection. J Med Virol. 2002;68: 378-83.

18. Shinoda K, Kobayashi A, Higashide T, et al. Detection of measles virus genomic RNA in tear samples from a patient with measles keratitis. Cornea. 2002;21:610-2.

19. Konjevoda S, Canovic S, Pastar Z, et al. Ophthalmic manifestations of novel coronaviruses: precautionary measures and diagnostic possibilities. J Glob Health. 2020;10:010430.

20. Hoffmann M, Kleine-Weber H, Schroeder S, et al. SARS-CoV-2 cell entry depends on ACE2 and TMPRSS2 and is blocked by a clinically proven protease inhibitor. Cell. 2020;181(271-280):e8.

21. Senanayake P, Drazba J, Shadrach K, et al. Angiotensin II and its receptor subtypes in the human retina. Investig Ophthalmol Vis Sci. 2007;48: 3301-11.

22. Sun Y, Liu L, Pan X, Jing M. Mechanism of action between the SARS-CoV S240 protein and the ACE2 receptor in eyes. Int J Ophthalmol. 2006;6:783-6.

23. Haddrell AE, Davies JF, Reid JP. Dynamics of particle size on inhalation of environmental aerosols and impact on deposition fraction. Environ Sci Technol. 2015;49:14512-21.

24. Clairfeuille T, Buchholz KR, Li Q, et al. Structure of the essential inner membrane lipopolysaccharidePbgA complex. Nature. 2020;584:479-83.
25. Bishop RE. The structure of a lipopolysaccharide regulator provides a route to new antibiotics. Nature. 2020;584:348-9.

26. Shigematsu S, Dublineau A, Sawoo O, et al. Influenza A virus survival in water is influenced by the species of origin of the host cell. Influenza Other Respir Viruses. 2014;8:123-30.

27. Harrison SC. Viral membrane fusion. Virology. 2015;479-480:498-507.

28. Blackie CA, Solomon JD, Scaffidi RC, Greiner JV, Lemp MA, Korb DR. The relationship between dry eye symptoms and lipid layer thickness. Cornea. 2009;28:789-94.

29. Eom Y, Lee JS, Kang SY, Kim HM. Correlation between quantitative measurements of tear film lipid layer thickness and meibomian gland loss in patients with obstructive meibomian gland dysfunction and normal controls. Am J Ophthalmol. 2013;155(1104-10):e2.

30. Güemes-Villahoz N, Burgos-Blasco B, Vidal-Villegas $B$, et al. Novel insights into the transmission of SARS-CoV-2 through the ocular surface and its detection in tears and conjunctival secretions: a review. Adv Ther. 2020;37:4086-95.

31. Bishop AH, Stapleton HL. Aerosol and surface deposition characteristics of two surrogates for Bacillus anthracis spores. Appl Environ Microbiol. 2016;82:6682-90.

32. Colbeck I, Lazaridis M. Aerosols and environmental pollution. Naturwissenschaften. 2010;97:117-31.

33. Ferron GA, Upadhyay S, Zimmermann R, Karg E. Model of the deposition of aerosol particles in the respiratory tract of the rat. II. Hygroscopic particle deposition. J Aerosol Med Pulm Drug Deliv. 2013;26:101-19.

34. Sanchez-Crespo A. Lung scintigraphy in the assessment of aerosol deposition and clearance. Semin Nucl Med. 2019;49(1):47-57.

35. Zhang T, Gao B, Zhou Z, Chang Y. Movement and deposition of PM2.5, in the upper respiratory tract for patients with heart failure: an elementary CFD study. Biomed Eng Online. 2016;15:138.

36. Sang X, Li Y, Yang L, et al. Lipid layer thickness and tear meniscus height measurements for differential diagnosis of evaporative dry eye subtypes. Int J Ophthalmol. 2018;11:1496-502. 
37. Chou YB, Fan NW, Lin PY. Lipid layer thickness and blinking pattern in patients with dry eye symptoms. Can J Ophthalmol. 2019;54:735-40.
38. Messmer EM. The pathophysiology, diagnosis, and treatment of dry eye disease. Dtsch Arztebl Int. 2015;112:71-81. 